\title{
Evaluation of influence of telmisartan on the pharmacokinetics and tissue distribution of canagliflozin in rats and mice
}

\author{
Xiao-Nan Wang ${ }^{1,2}$, Ying Li $^{2}$, Lu Meng ${ }^{3}$, Cong-Yang Ding ${ }^{2}$, Zhan-Jun Dong ${ }^{1,2}$ \\ ${ }^{1}$ Graduate School of Hebei Medical University, Shijiazhuang, China; ${ }^{2}$ Department of Pharmacy, Hebei General Hospital, Shijiazhuang, China; ${ }^{3}$ The \\ Second Hospital of Shijiazhuang, Shijiazhuang, China \\ Contributions: (I) Conception and design: ZJ Dong, XN Wang; (II) Administrative support: ZJ Dong; (III) Provision of study materials or patients: \\ XN Wang; (IV) Collection and assembly of data: XN Wang, Y Li, L Meng, CY Ding; (V) Data analysis and interpretation: XN Wang, Y Li; \\ (VI) Manuscript writing: All authors; (VII) Final approval of manuscript: All authors. \\ Correspondence to: Zhan-Jun Dong. Graduate School of Hebei Medical University, Shijiazhuang, China; Department of Pharmacy, Hebei General \\ Hospital, Shijiazhuang, China. Email: dzjhbgh@163.com.
}

\begin{abstract}
Background Canagliflozin, a sodium glucose cotransporter 2 (SGLT2) inhibitor, is widely used for the treatment of type 2 diabetes mellitus. However, drug interactions with canagliflozin can affect its glucoselowering therapeutic effects or exacerbate its adverse effects. Telmisartan, an angiotensin receptor blocker (ARB), has been approved for the treatment of diabetic kidney disease. This study aimed to investigate the effects of telmisartan on the pharmacokinetics and tissue distribution of canagliflozin.

Methods: An ultra-performance liquid chromatography-tandem mass spectrometry method was successfully validated to determine the levels of canagliflozin in the plasma and tissues. The main pharmacokinetic parameters were calculated using the non-compartmental model.

Results: Compared with animals administered canagliflozin alone, the area under the receiver operating characteristic curve of animals co-administered telmisartan and canagliflozin was significantly increased after a single-day administration, but significantly decreased after a seven-day treatment regimen (both $\mathrm{P}<0.05$ ). The highest concentrations of canagliflozin were detected in the kidneys, followed by the intestine, liver, heart, lung, spleen, and brain tissues. Furthermore, the concentration of canagliflozin in the heart, liver, lung, and kidney tissues at 2 hours post-administration was significantly higher in the telmisartan and canagliflozin group compared to the group treated with canagliflozin alone $(\mathrm{P}<0.05)$.
\end{abstract}

Conclusions: A pharmacokinetic drug-drug interaction between telmisartan and canagliflozin might occur during drug co-administration.

Keywords: Telmisartan; canagliflozin; pharmacokinetics; tissue distribution; drug-drug interaction

Submitted Dec 16, 2020. Accepted for publication Mar 11, 2021.

doi: 10.21037/apm-21-65

View this article at: http://dx.doi.org/10.21037/apm-21-65

\section{Introduction}

Diabetes is associated with a high risk of cardiovascular and renal diseases $(1,2)$. The increased incidence of diabetes in the past decades has become the major cause of end-stage kidney disease (3). Telmisartan, an angiotensin receptor blocker (ARB), is one of the most frequently used drugs for the treatment of hypertension. ARBs were first shown to be effective 19 years ago, and they are the only approved treatment for renoprotection in patients with type 2 diabetes
(4,5). Telmisartan is a substrate of $\mathrm{P}$-glycoprotein (P-gp/ $\mathrm{ABCB} 1$ ), breast cancer resistance protein (BCRP/ABCG2), and multidrug resistance-associated protein 2 (MRP2/ $\mathrm{ABCC} 2$ ). Specifically, telmisartan is a potent inhibitor of $\mathrm{P}-\mathrm{gp}$ and a moderate inhibitor of MRP2 and BCRP $(6,7)$.

Canagliflozin reduces blood glucose levels independently of insulin secretion via inhibition of the sodium glucose cotransporter 2 (SGLT2) receptors in the proximal renal tubules, leading to decreased renal reabsorption of glucose. 
Furthermore, SGLT2 inhibitors are a novel class of drugs that have been shown to be effective for the treatment of renal diseases, as demonstrated in the Canagliflozin and Renal Events in Diabetes with Established Nephropathy Clinical Evaluation (CREDENCE) and the Dapagliflozin and Prevention of Adverse outcomes in Chronic Kidney Disease (DAPA-CKD) clinical trials $(8,9)$, both clinical trials required the participates to receive treatment with angiotensin-converting enzyme inhibitors (ACEIs) or ARBs, the combination of SGLT2 inhibitors and ARBs is a promising treatment therapy in renal disease, while the potential interactions have not been investigated. Considering that canagliflozin is a substrate of the three efflux transporters (10), modulation of P-gp, BCRP, and MRP2 activities by telmisartan may cause significant changes in the pharmacokinetic profile of canagliflozin. Compared to rats, mice have a higher amino acid homology of transporter proteins to humans (11-13), however, the pharmacokinetic experiment itself has many plasma volume, then we chose rats in consideration of animal welfare regulations for mice. For tissue distribution and subsequent molecular mechanism, we still chose mice as the experiment subjects.

Both SGLT2 inhibitors and ARBs act on tubuloglomerular feedback (TGF), with the former altering afferent arteriole vasoconstriction, while the latter changes efferent arteriole vasodilation $(14,15)$. However, it remains unknown whether combined SGLT2 and ARB inhibition can normalize the intraglomerular hypertension and hyperfiltration associated with renal protection.

It is widely accepted that the concentration of a drug in the tissues is fundamental in determining its pharmacological effects and adverse effects. This study aimed to investigate whether co-administration of telmisartan alters the pharmacokinetics of canagliflozin. The plasma and tissue distribution of canagliflozin were assessed. The results provide clinical guidance regarding adverse drug reactions during co-administration of telmisartan and canagliflozin. We present the following article in accordance with the ARRIVE reporting checklist (available at http://dx.doi.org/10.21037/apm-21-65).

\section{Methods}

\section{Chemicals and reagents}

Canagliflozin (standard, purity $\geq 98 \%$ ) was obtained from Shanghai Zhen Zhun Biological Technology Co., Ltd.
(Shanghai, China), while canagliflozin (raw material, purity $\geq 99 \%$ ) was purchased from Shanghai Macklin Biochemical Co., Ltd. (Shanghai, China). Empagliflozin (standard, purity $\geq 99 \%$ ) was obtained from ApexBio (Houston, TX, USA) and telmisartan (raw material, purity $\geq 99 \%$ ) was purchased from Aladdin Co., Ltd. (Shanghai, China). High performance liquid chromatography (HPLC)-grade methyl tert-butyl ether and formic acid were obtained from Fisher Scientific Ltd. (Pittsburgh, PA, USA). Ultrapure water was obtained from Wahaha Group Co., Ltd. (Hangzhou, China).

\section{Apparatus and conditions}

The ultra-performance liquid chromatography-tandem mass spectrometry (UPLC-MS/MS) system consisted of a Shimadzu Nexera LC-30A UPLC system (Shimadzu, Kyoto, Japan) with a Waters BEH C18 column $(2.1 \mathrm{~mm} \times$ $100 \mathrm{~mm}, 1.7 \mu \mathrm{m}$; Waters Co., Ltd., Dublin, Ireland) and an API 5500 triple-quadrupole mass spectrometer (Sciex Co., Ltd., Framingham, MA, USA).

The optimized method used binary gradient mobile phases with water (containing $0.1 \%$ formic acid) as mobile phase $\mathrm{A}$, and acetonitrile as mobile phase $\mathrm{B}$. The gradient program was applied as follows: phase B was initially balanced at $40 \%$, then linearly increased from $40 \%$ to $75 \%$ for 4 minutes, and slightly increased from $75 \%$ to $85 \%$ in 0.8 minutes. The pre-column equilibrium time was 1 minute and the flow rate was $0.25 \mathrm{~mL} / \mathrm{min}$. The prepared sample $(3 \mu \mathrm{L})$ was injected into the UPLC-MS/MS system. The auto-sampler and column temperature were maintained at 4 and $40{ }^{\circ} \mathrm{C}$, respectively.

The mass spectrometer was equipped with an electrospray ionization (ESI) source. The multiple reaction monitoring (MRM) analysis in the positive ion ESI mode was conducted by monitoring the ion transitions from $\mathrm{m} / \mathrm{z}$ 462.2 to 191.0 for canagliflozin, and $\mathrm{m} / \mathrm{z} 451.4$ to 71.0 for empagliflozin (internal standard, IS), with the declustering potential set at 66.5 and $66 \mathrm{~V}$, and collision energy at 35.1 and $20 \mathrm{eV}$, for canagliflozin and IS, respectively.

\section{Animals}

Male Sprague-Dawley rats $(220 \pm 20 \mathrm{~g})$ and male Kunming mice $(26 \pm 2 \mathrm{~g})$ were purchased from the Experimental Animal Center of Hebei Medical University (Shijiazhuang, China). Animal were housed in an environmentally controlled room (temperature: $25 \pm 2{ }^{\circ} \mathrm{C}$, humidity: $55 \% \pm 5 \%, 12$-hour dark-light cycle), and given free access 
to water and food. The animals were fasted for 8 hours prior to the experiments, with free access to drinking water during the experiments. This study was approved by the Hebei General Hospital Ethics Committee (No.202103). All processes were performed according to the Guidelines for the Care and Use of Laboratory Animals of the Japanese Pharmacological Society.

\section{Drug administration and sample collection}

In the pharmacokinetic studies, Sprague-Dawley rats were randomly divided into four groups (each group consisting of six replicates). Group A and B animals were administered canagliflozin with and without telmisartan for a single day, respectively. Animals in group C and D were administered canagliflozin with and without telmisartan for seven consecutive days, respectively. Compounds for oral gavage were mixed in $0.5 \%$ sodium carboxymethyl cellulose (CMC-Na) solution. The administered doses were $10 \mathrm{mg} / \mathrm{kg}$ for canagliflozin and $8 \mathrm{mg} / \mathrm{kg}$ for telmisartan. Blood samples $(0.2 \mathrm{~mL})$ were collected via the orbital vein in heparin-containing capillary tubes at $0,1,1.5,2,3,4,5$, $6,8,10,12,24$, and 48 hours after drug administration and centrifuged at 3,500 rpm for 10 minutes. The supernatant was transferred into a new tube and stored at $-80^{\circ} \mathrm{C}$.

For the tissue distribution studies, the Kunming mice were randomly separated into three groups. These groups were given oral administration of $0.5 \% \mathrm{CMC}-\mathrm{Na}$, canagliflozin $(15 \mathrm{mg} / \mathrm{kg})$, or canagliflozin $(15 \mathrm{mg} / \mathrm{kg})$ plus telmisartan $(12 \mathrm{mg} / \mathrm{kg})$ for seven consecutive days. The heart, liver, spleen, lung, kidney, intestine, and brain tissues were collected at 0, 2, 4, 6, 8, 10, and 24 hours (three replicates at each time point). All tissues were washed with saline, dried using filter paper, and stored at $-80^{\circ} \mathrm{C}$.

\section{Preparation of standards and quality control samples}

Stock solutions of canagliflozin and IS were prepared in methanol at $1 \mathrm{mg} / \mathrm{mL}$. The canagliflozin and IS working solutions were prepared by diluting the stock solutions with methanol. Calibration standard samples were prepared by spiking a working solution of canagliflozin and IS with the plasma/tissue homogenates, followed by an extraction process to yield calibration standards of 50, 200, 1,000, $5,000,10,000,15,000,20,000$, and 30,000 ng/mL. The low (LQC), medium (MQC), and high (HQC) quality control samples $(10,800$, and $2,400 \mathrm{ng} / \mathrm{mL}$, respectively) and lower limit of quantification (LLOQ, $5 \mathrm{ng} / \mathrm{mL}$ ) were considered as the standards.

\section{Sample preparation}

A liquid-liquid extraction (LLE) procedure was performed to extract canagliflozin from plasma and tissue homogenates. Plasma samples $(50 \mu \mathrm{L})$ were combined with $5 \mu \mathrm{L}$ of IS and $200 \mu \mathrm{L}$ of methyl tert-butyl ether, vortexed for 1 minute and centrifuged for 10 minutes at $12,000 \mathrm{rpm}$. The supernatant was transferred to a new centrifuge tube, and evaporated till dry under a stream of nitrogen. The residue was reconstituted in the initial mobile phase, centrifuged for 2 minutes at $12,000 \mathrm{rpm}$, and $50 \mu \mathrm{L}$ of the solution was transferred into sample vials.

Tissue samples weighing $100 \mathrm{mg}$ (except for the spleen, which in total weighed less than $100 \mathrm{mg}$ ) were homogenized with saline $(1: 2 \mathrm{w} / \mathrm{v}$ for the spleen, and 1:1 w/v for all other tissues) for 1 minute. The supernatant was transferred to a new centrifuge tube and processed as described above for the plasma samples.

\section{Method validation}

The analytical method for evaluating canagliflozin in the plasma and tissue homogenate samples using UPLCMS/MS was validated for selectivity, sensitivity, linearity, accuracy, precision, extraction recovery, matrix effect, and stability based on the Bioanalytical Method Validation guidelines published by the United States Food and Drug Administration (USFDA) (16,17). The method was fully validated for plasma and liver homogenates (representative of tissue homogenates), but was only validated for selectivity, linearity, accuracy, and precision for all other tissue homogenates.

Selectivity was evaluated by comparing the chromatograms of six different batches of blank samples with the corresponding spiked biological samples. The signal-tonoise $(\mathrm{S} / \mathrm{N})$ ratios of the LLOQ should be no less than 10:1. Biological samples were quantified using the peak area ratios of canagliflozin to IS. A standard curve in the form of $y=a x+$ b was used, where $x$ represents canagliflozin concentration in the sample and $y$ represents the ratio of canagliflozin peak area to that of IS. A linear least square regression analysis was performed to determine the slopes, intercepts, and coefficients $\left(\mathrm{r}^{2}\right)$, and demonstrate the linearity of this method. The calibration curves were analyzed on three consecutive days.

The intra-day accuracy and precision were confirmed by 
six replicates of four concentrations of the quality control (QC) samples, while the inter-day accuracy and precision were validated by six replicates of four levels of QC samples on three consecutive days. The accuracy was expressed by [(mean observed concentration $) /($ spiked concentration $)] \times$ $100 \%$. The precision was calculated by relative standard deviation (RSD). Variation was acceptable up to $\pm 15 \%$, and the LLOQ up to $\pm 20 \%$.

Quantitative assessment of the matrix effect was performed by post extraction spiking. The peak area (A) of the standard analyte in spiked blank plasma/liver homogenates was compared with the corresponding peak area (B) obtained by adding the standard analyte in the initial mobile phase at concentrations of 10,800 , and $2,400 \mathrm{ng} / \mathrm{mL}$ for canagliflozin in six replicates. The peak area ratio of $\mathrm{A} / \mathrm{B}$ was shown as a quantitative measure of the matrix effect. Extraction recoveries were calculated by comparing the mean peak area of spiked plasma/liver homogenates to those of analytes in the matrix by spiking extracted analyte-free plasma/liver homogenates prior to chromatography in six replicates of three levels of QC samples. The matrix effect and extraction recovery of the IS were calculated using a single concentration $(200 \mathrm{ng} / \mathrm{mL})$ and the same procedure as that for canagliflozin.

The stability of plasma/liver homogenates under different storage conditions was evaluated at the levels of LQC, MQC, and HQC samples $(\mathrm{n}=6)$. Short-term stability was determined in an autosampler at $4{ }^{\circ} \mathrm{C}$ for 24 hours and at room temperature $\left(25^{\circ} \mathrm{C}\right)$ for 24 hours. Long-term stability was determined in the samples after storage at $-80{ }^{\circ} \mathrm{C}$ for 30 days. The QC samples at $-80{ }^{\circ} \mathrm{C}$ were kept at room temperature, and repeatedly thawed in three cycles to determine the freeze-thaw stability of the analytes.

\section{Data analysis}

The acquisition software used were Analyst TF 1.7.1 software and Analyst software (AB Sciex, Redwood city, CA, USA). The pharmacokinetic and tissue homogenate parameters were processed by non-compartmental analysis using DAS 2.1.1 software (Mathematical Pharmacology Professional Committee of China, Shanghai, China). Data were presented as mean \pm standard deviation $(\mathrm{SD})$, no data was excluded in pharmacokinetic and tissue distribution studies. Statistical differences were analyzed by Student's $t$-test and nonparametric rank-sum test with a $\mathrm{P}$ value $<0.05$ indicating a statistically significant difference (SPSS 25.0 software, SPSS Inc., Chicago, IL, USA).

\section{Results}

\section{Method validation}

The selectivity was determined by comparing the typical MRM chromatograms of blank plasma/liver homogenates, blank plasma/liver homogenates spiked with canagliflozin and IS, and plasma/liver homogenates after the oral administration of canagliflozin (Figure 1). Empagliflozin was used as the IS. Empagliflozin and canagliflozin were detected at 2.14 and 3.52 minutes, respectively. No endogenous interference was observed in the plasma/liver homogenates. Regression equations and correlation of coefficients for canagliflozin in plasma and tissue homogenates are shown in Table 1. The calibration curves of the correlation coefficients $\left(r^{2}\right)$ values were more than 0.99 , the linear range was 5 $3,000 \mathrm{ng} / \mathrm{mL}$, and the LLOQ for canagliflozin was $5 \mathrm{ng} / \mathrm{mL}$.

The intra-day and inter-day accuracy and precision values in plasma and liver homogenates are shown in Table 2. These results demonstrated that this method was an accurate and reproducible technique for detecting canagliflozin in plasma and liver homogenates.

The results for the matrix effect and extraction recovery values in plasma and liver homogenates are shown in Table 3. The matrix effect varied from $90.18 \%$ to $98.64 \%$, indicating that there were no endogenous substances or any interference with canagliflozin determination in plasma and liver homogenates. The extraction recovery in different matrices ranged from $91.73 \%$ to $107.61 \%$.

The results of the stability studies in plasma and liver homogenates under different conditions are summarized in Table 4 . The RSD values were less than $15 \%$ for all analytes. These results indicated that canagliflozin was stable in plasma and liver homogenates that were stored at $-80^{\circ} \mathrm{C}$ for 30 days and further subjected to three freeze-thaw cycles (each at $-80{ }^{\circ} \mathrm{C}$ for 24 hours). In addition, the processed plasma and liver homogenate samples were stable in the autosampler at $4{ }^{\circ} \mathrm{C}$ for 24 hours, and at room temperature for 24 hours.

\section{Pharmacokinetic studies}

The validated method was successfully applied to examine the concentration of canagliflozin in rat plasma after administration by oral gavage. The mean plasma concentration-time curves of canagliflozin after single- 
A cps
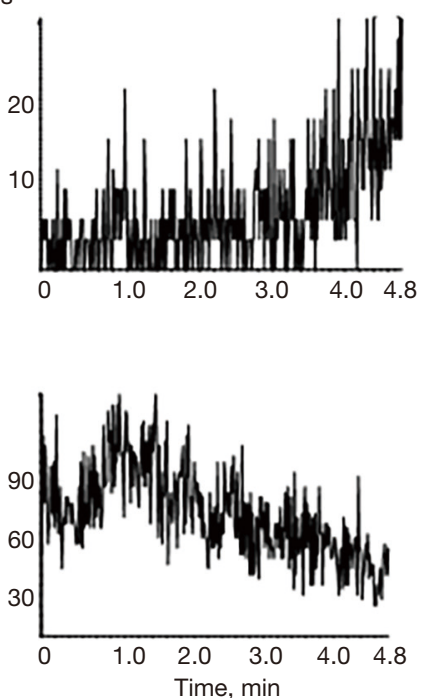

cps
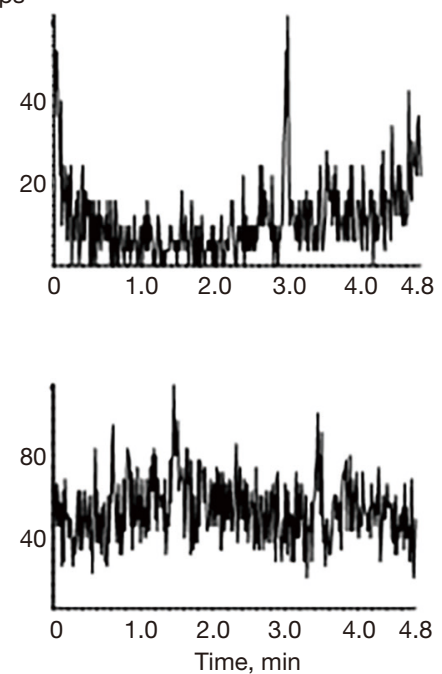

B
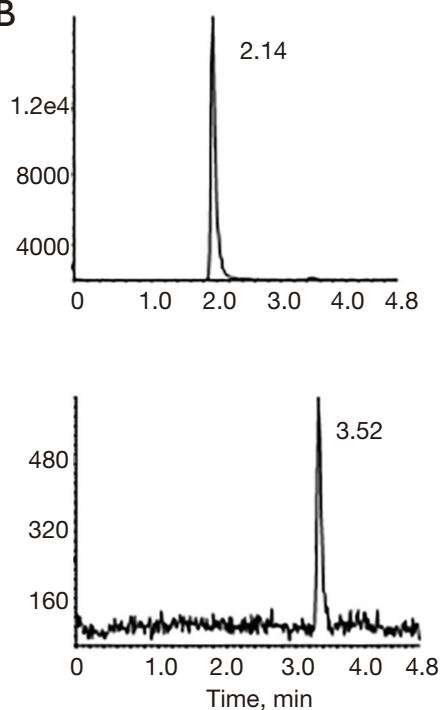

E
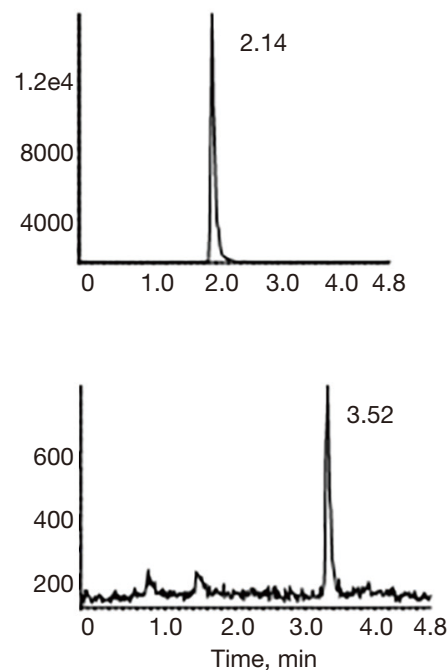

C
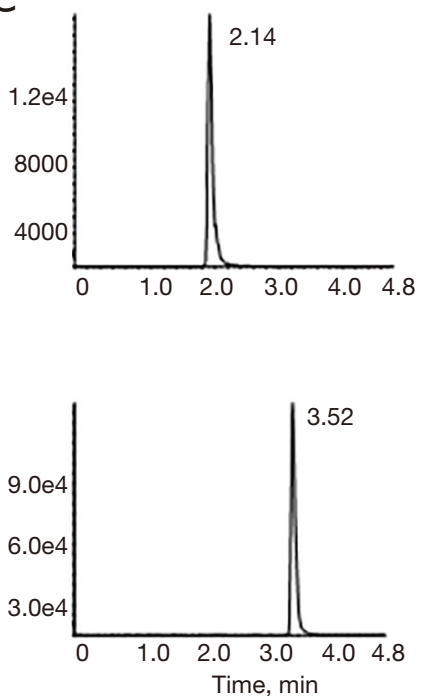

$\mathrm{F}$
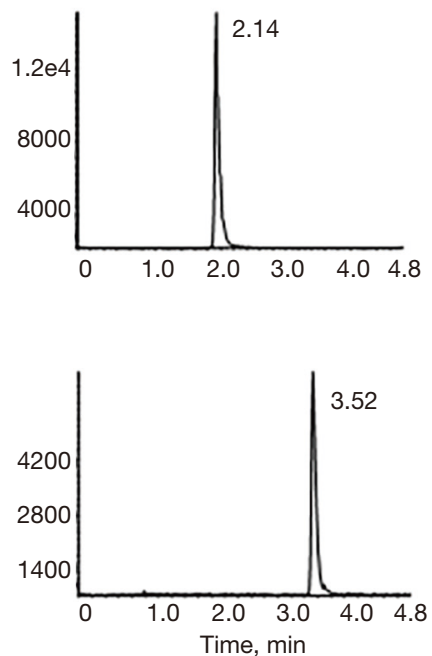

Figure 1 Chromatograms of internal standard (IS) and canagliflozin in plasma and liver homogenates. (A) Blank plasma, (B) blank plasma spiked with IS and canagliflozin, (C) rat plasma at 1 hour after oral administration canagliflozin spiked with IS, (D) blank liver homogenates, (E) blank liver homogenates spiked with IS and canagliflozin, (F) mice liver homogenates at 2 hours after oral administration of canagliflozin spiked with IS.

and seven-day administration are shown in Figure 2. The primary pharmacokinetic parameters were determined with the DAS 2.1.1 software using the non-compartmental model and the results are listed in Table 5 .

Compared with animals treated with canagliflozin alone, animals co-administered telmisartan and canagliflozin had slightly higher concentrations of canagliflozin in the plasma after a single-day administration, however, this was not statistically significant. The area under the curve of 0 to 48 hours $\left(\mathrm{AUC}_{0-48}\right)$ and the area under the curve of 0 to infinity $\left(\mathrm{AUC}_{0-\infty}\right)$ of canagliflozin in the combined treatment group were significantly increased by $57.7 \%$ and $56.6 \%$, respectively, while the apparent volume of distribution divided by the absorption fraction $\left(\mathrm{V}_{\mathrm{z}} / \mathrm{F}\right)$ and the clearance divided by the absorption fraction $\left(\mathrm{CL}_{z} / \mathrm{F}\right)$ significantly decreased by $41.7 \%$ and $31.1 \%$, respectively. The half time $\left(\mathrm{t}_{1 / 2}\right)$ 
Table 1 The calibration curves, coefficient, range, and LLOQ of canagliflozin in different matrices

\begin{tabular}{|c|c|c|c|c|}
\hline Matrix & Calibration curve & Coefficient & Range (ng/mL) & LLOQ $(\mathrm{ng} / \mathrm{mL})$ \\
\hline Heart & $y=0.00518 x+0.0044$ & 0.9945 & $5-3,000$ & 5 \\
\hline Liver & $y=0.00587 x+0.0165$ & 0.9990 & $5-3,000$ & 5 \\
\hline Spleen & $y=0.00638 x+0.0383$ & 0.9978 & $5-3,000$ & 5 \\
\hline Kidney & $y=0.00637 x+0.0752$ & 0.9974 & $5-3,000$ & 5 \\
\hline Intestine & $y=0.00739 x+0.0171$ & 0.9988 & $5-3,000$ & 5 \\
\hline Brain & $y=0.00745 x+0.0202$ & 0.9946 & $5-3,000$ & 5 \\
\hline
\end{tabular}

LLOQ, lower limit of quantification.

Table 2 The accuracy and precision values for the analysis of canagliflozin in plasma and liver homogenates

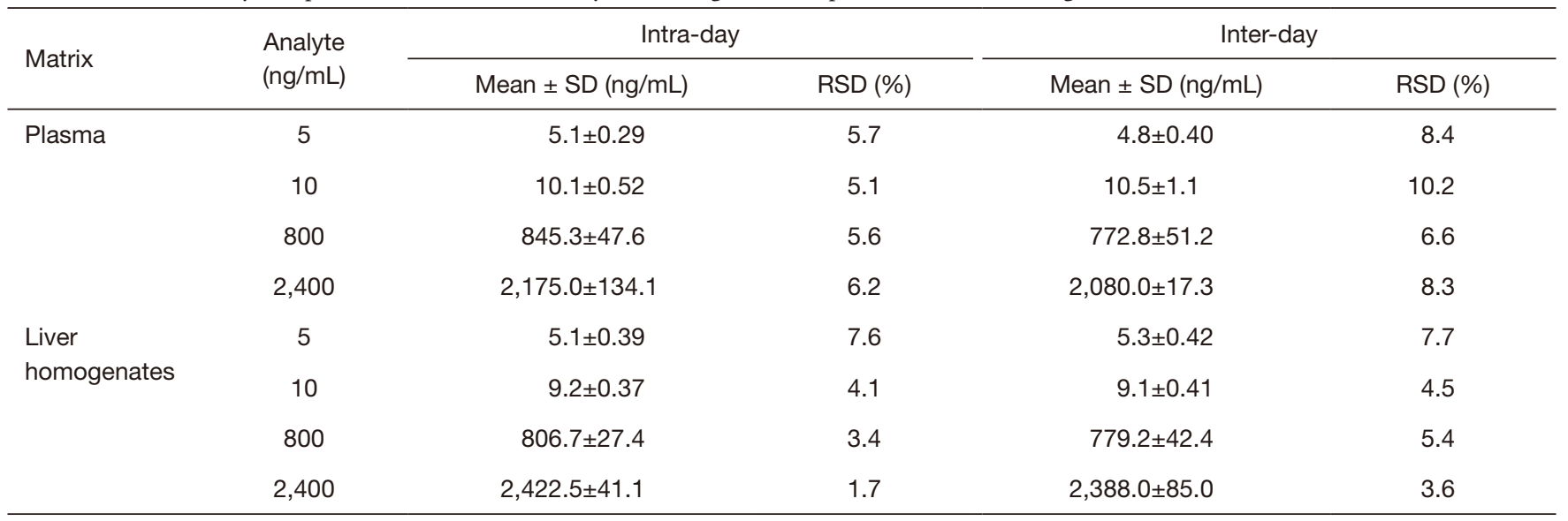

$\mathrm{SD}$, standard deviation; RSD, relative standard deviation.

Table 3 The matrix effects and extraction recoveries of canagliflozin and the IS in plasma and liver homogenates

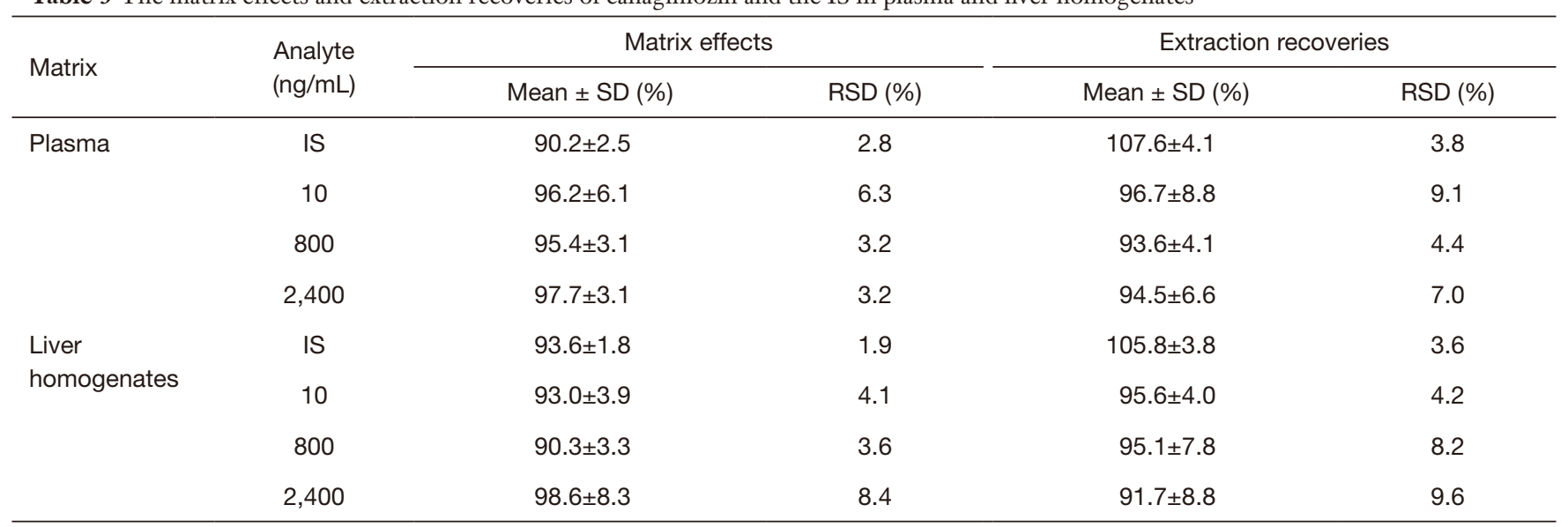

IS, internal standard; SD, standard deviation; RSD, relative standard deviation. 
Table 4 The stability of canagliflozin in plasma and liver homogenates

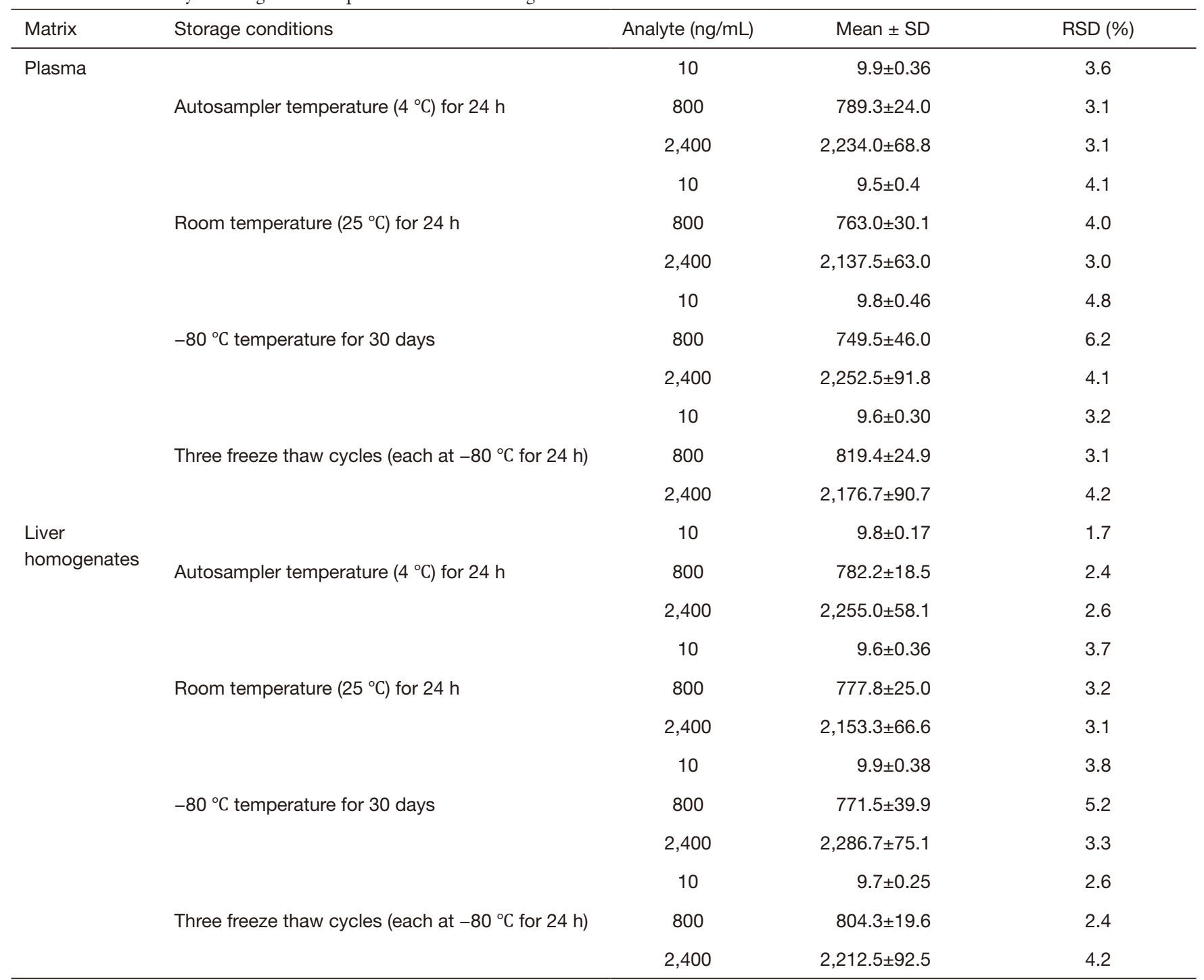

SD, standard deviation; RSD, relative standard deviation.
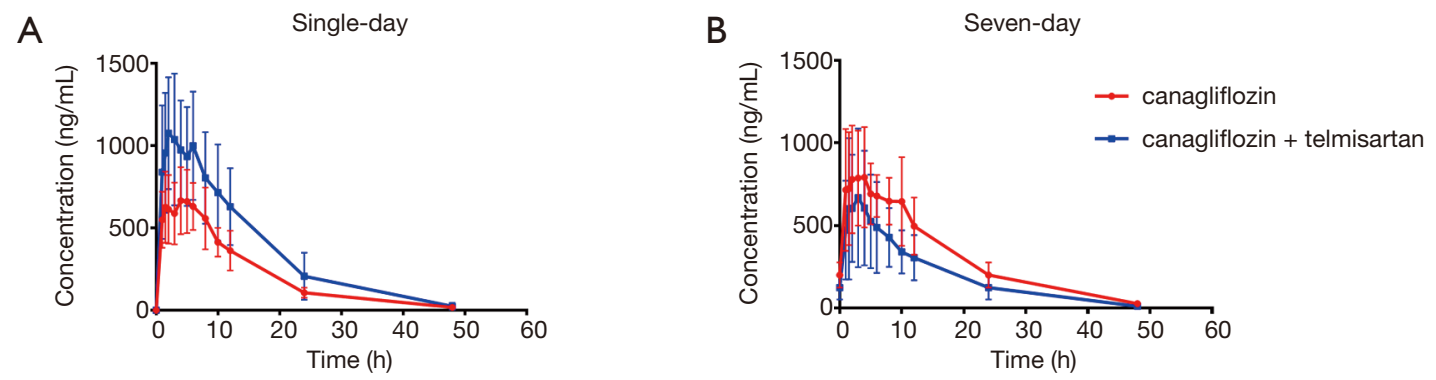

Figure 2 The mean plasma concentration-time curves for canagliflozin with or without telmisartan. (A) After single-day administration; (B) after seven-day administration. Data are expressed as mean \pm standard deviation, $\mathrm{n}=6$. 
Table 5 The effects of telmisartan on the pharmacokinetic parameters of canagliflozin in rat plasma

\begin{tabular}{|c|c|c|c|c|}
\hline \multirow{2}{*}{ Parameters (unit) } & \multicolumn{2}{|c|}{ Single-day administration } & \multicolumn{2}{|c|}{ Seven-day administration } \\
\hline & Canagliflozin & Canagliflozin + telmisartan & Canagliflozin & Canagliflozin + telmisartan \\
\hline $\mathrm{AUC}_{0-\mathrm{t}}(\mathrm{ng} / \mathrm{L} \times \mathrm{h})$ & $10,579.5 \pm 2,834.9$ & $16,685.2 \pm 6,215.0^{*}$ & $14,979.1 \pm 3,401.7$ & $9,289.5 \pm 3,762.7^{*}$ \\
\hline MRT (h) & $12.4 \pm 1.0$ & $11.9 \pm 2.9$ & $13.2 \pm 1.8$ & $13.1 \pm 4.8$ \\
\hline$t_{1 / 2 z}(h)$ & $8.4 \pm 1.1$ & $7.2 \pm 1.8^{*}$ & $8.4 \pm 0.81$ & $8.5 \pm 3.1$ \\
\hline $\mathrm{V}_{\mathrm{z}} / \mathrm{F}(\mathrm{L} / \mathrm{kg})$ & $11,755.7 \pm 2,933.9$ & $6,856.5 \pm 2,677.0^{*}$ & $8,278.3 \pm 1,964.2$ & $13,655.3 \pm 5,940.3$ \\
\hline $\mathrm{CL}_{\mathrm{z}} / \mathrm{F}(\mathrm{L} / \mathrm{h} / \mathrm{kg})$ & $979.2 \pm 244.7$ & $674.6 \pm 271.5^{\star}$ & $684.4 \pm 163.1$ & $1113.9 \pm 318.1^{*}$ \\
\hline $\mathrm{C}_{\max }(\mathrm{ng} / \mathrm{L})$ & $749.8 \pm 190.0$ & $1,109.3 \pm 377.7$ & $993.1 \pm 235.5$ & $679.2 \pm 410.4$ \\
\hline
\end{tabular}

Data are shown in mean \pm standard deviation, $n=6,{ }^{*} \mathrm{P}<0.05$ compared with the corresponding canagliflozin group. $\mathrm{AUC}_{0-\mathrm{t}}$, area under the curve of 0 to $t ; A U_{0-\infty}$, area under the curve of 0 to infinity; MRT, mean residence time of 0 to infinity; $t_{1 / 2 z}$, half time; Tmax, peak time; $V z / F$, apparent volume of distribution divided by absorption fraction; CLz/F, clearance divided by absorption fraction; Cmax, peak concentration.

significantly reduced by $14.3 \%$. In contrast, after a seven-day drug administration, the plasma concentrations of canagliflozin were slightly, but not significantly, decreased in the combined treatment group. The $\mathrm{AUC}_{0-48}$ and $\mathrm{AUC}_{0-\infty}$ of canagliflozin significantly decreased by $38 \%$ and $36.1 \%$, respectively, while the $\mathrm{CL}_{z} / \mathrm{F}$ significantly increased by $62.9 \%$.

\section{Tissue distribution study}

The validated method was also successfully applied to examine the concentration of canagliflozin in mice tissues after oral gavage administration. The tissue distribution of canagliflozin was detected at $0,2,4,6,8,10$, and 24 hours after a seven-day administration in the presence or absence of telmisartan. Canagliflozin was widely distributed in all collected tissues, as shown in Figure 3. After a seven-day administration, the highest concentrations of canagliflozin were detected in the kidneys, followed by the intestine, liver, lung, heart, spleen, and brain, for both groups. In the canagliflozin group, the concentration of canagliflozin showed an obvious double-peak phenomenon, at approximately 4 and 8 hours. However, in the combined treatment group, the concentration of canagliflozin showed a peak at 2 hours. Furthermore, the concentrations of canagliflozin in the heart, liver, lung, and kidney tissues at 2 hours were significantly higher in the combined treatment group compared to the group treated with canagliflozin alone. Moreover, the concentration of canagliflozin in all collected tissues at 10 and 24 hours were slightly higher in the combined treatment group compared to the group treated with canagliflozin alone.

\section{Discussion}

The UPLC-MS/MS method was successfully applied to study the influence of telmisartan on the pharmacokinetics and tissue distribution of canagliflozin. This LLE approach was used to process the biological samples due to its higher extraction recovery compared to that of previous LLE approaches and protein precipitation methods (18-20). Different LLE solvents, including ethyl acetate, dichloromethane, and methyl tert-butyl ether, were explored. Finally, methyl tert-butyl ether was chosen as the solvent as it provided higher extraction recovery and was only required in low volumes (20-22).

In the pharmacokinetics studies, compared with the group treated with canagliflozin alone, the $\mathrm{AUC}_{0-48}$ and $\mathrm{AUC}_{0-\infty}$ of canagliflozin in the combined treatment group significantly increased by $57.7 \%$, and $56.6 \%$, respectively, while, the $\mathrm{t}_{1 / 2}, \mathrm{~V}_{z} / \mathrm{F}$, and $\mathrm{CL}_{z} / \mathrm{F}$ significantly decreased, after singleday administration. However, the plasma concentrations of canagliflozin were slightly, but not significantly, decreased in the combined treatment group after the seven-day administration, thereby implying a higher concentration of canagliflozin in tissues with rich blood supply compared to plasma. The $\mathrm{AUC}_{0-48}$ and $\mathrm{AUC}_{0-\infty}$ significantly decreased by $38 \%$ and $36.1 \%$, respectively, while $\mathrm{CL}_{z} / \mathrm{F}$ significantly increased by $62.9 \%$ after the seven-day administration. Telmisartan has low water-solubility and is mainly absorbed through the intestines (23). The decreased plasma 


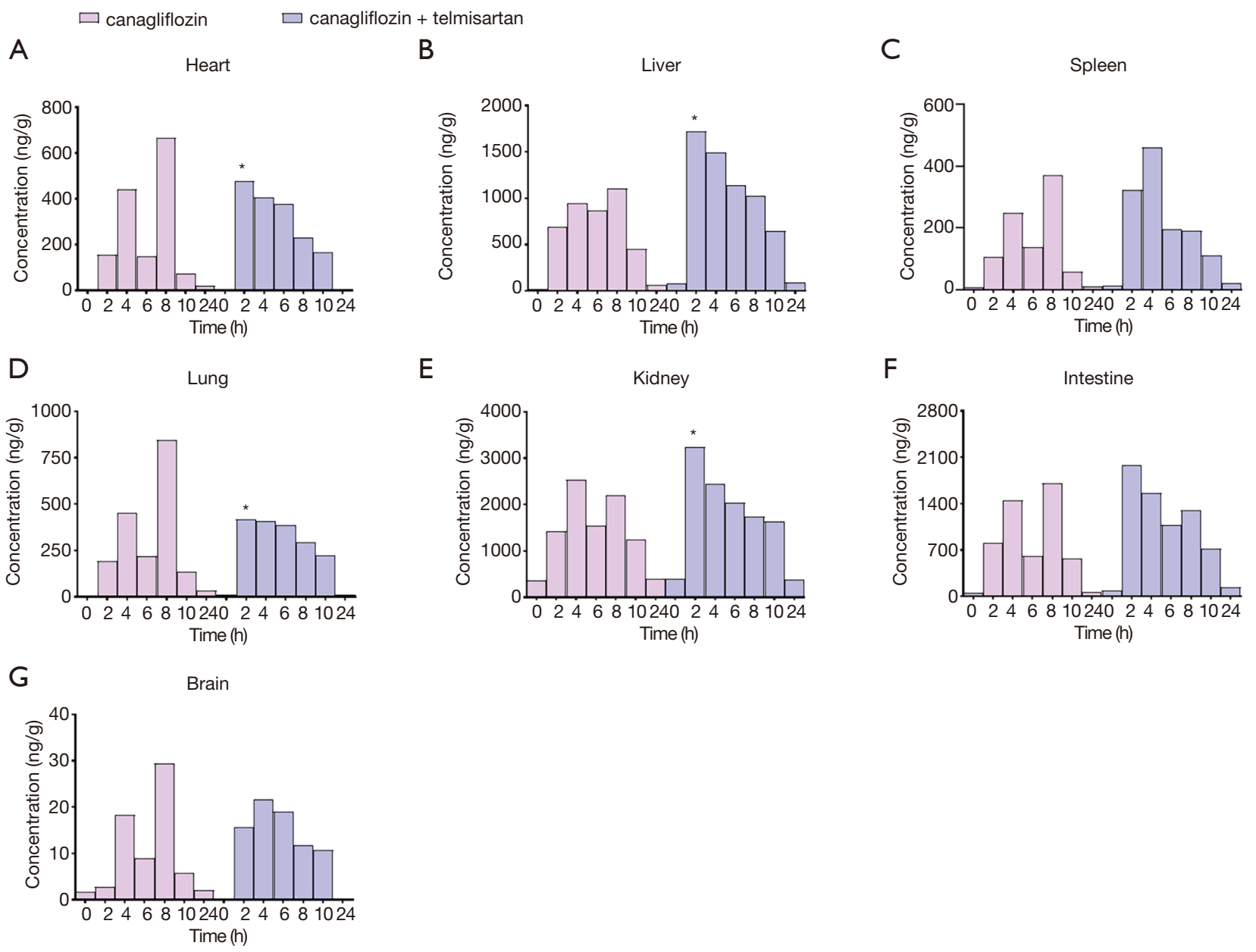

Figure 3 The mean concentrations of canagliflozin in different matrices at different time points after seven-day administration of canagliflozin with or without telmisartan. (A) Heart, (B) liver, (C) spleen, (D) lung, (E) kidneys, (F) intestine, (G) brain. * ${ }^{*}<0.05$, compared with the corresponding canagliflozin group, data are expressed as mean \pm standard deviation, $\mathrm{n}=3$.

concentrations of canagliflozin may result from a direct inhibition of canagliflozin absorption from the gastrointestinal tract mediated by the long-term use of telmisartan.

For the tissue distribution studies, in accordance with the USFDA guidelines, at least three time points were examined to determine the drug distribution in the absorption phase, equilibrium phase, and elimination phase. Similar to the previous rat plasma concentration-time curves in the pharmacokinetic studies, mice were sacrificed at $0,2,4,6,8,10$, and 24 hours after the seven-day administration of canagliflozin.

After administration of canagliflozin for seven days, the highest concentrations of the drug were detected in the kidneys, followed by the intestine, liver, lung, heart, spleen, and brain tissues, which was consistent with a previous tissue distribution study in mice after intragastric administration of canagliflozin $(24,25)$. The concentration of canagliflozin in the kidneys and liver tissues were higher than that in other collected tissues at the corresponding time points. This suggested that metabolism and excretion were mainly facilitated by the liver and kidneys. High concentrations of canagliflozin were observed in the intestinal tissues, however, its metabolites were not detected, suggesting little metabolism of canagliflozin in the gastrointestinal tract (26). Additionally, canagliflozin was poorly absorbed in the brain, suggesting that it cannot effectively cross the blood-brain barrier. Furthermore, except for the kidneys, canagliflozin was cleared from all the tissues at 24 hours, and no accumulation was observed in the collected tissues. These results implied that the kidneys were the target organ for canagliflozin.

The concentrations of canagliflozin in the heart, liver, 
lung, and kidney tissues at 2 hours in the combined treatment group were significantly higher than that in the group treated with canagliflozin alone. The concentrations of canagliflozin in all the collected tissues at the elimination phase in the combined treatment group were slightly higher compared to animals treated with canagliflozin alone, and no significant adverse effect was observed. While ABC transporters have been shown to play an important role in the pharmacokinetics of drugs in vitro, its impact in vivo is yet to be explored. There is clear evidence that in vivo telmisartan is a substrate and potent inhibitor of $\mathrm{P}-\mathrm{gp} / \mathrm{ABCB} 1$, and a moderate inhibitor of MRP2/ABCC2 and BCRP/ABCG2. Similarly, in vitro data indicated that canagliflozin is also a substrate of all three efflux transporters. It is possible that telmisartan, being a drug of long-term use, may alter the expression of these efflux transports, and thereby influence the pharmacokinetics of canagliflozin in vivo. However, the detailed mechanisms are yet to be fully elucidated.

There are no related reports of canagliflozin and telmisartan binding as new compounds in vivo. Glucuronic acid binding is the mainly metabolic form of telmisartan (27); M5 and M7 were the O-glucuronide metabolite of canagliflozin, which were two main inactive metabolites $(21,28)$.

In this report, a UPLC-MS/MS method was successfully validated for determining the concentrations of canagliflozin in plasma and tissue homogenates. Telmisartan increased the AUC of canagliflozin in rat plasma after a single-day administration, but decreased the AUC of canagliflozin in rat plasma after a seven-day administration regimen. Canagliflozin was widely distributed in all tissues collected, while telmisartan significantly increased the concentration of canagliflozin in the heart, liver, lung, and kidney tissues at 2 hours after the seven-day administration. These results suggested that a pharmacokinetic drug-drug interaction between telmisartan and canagliflozin might occur when they are co-administered in rats and mice, animals and humans do have species differences, the result we get need to be validated in humans.

\section{Acknowledgments}

Funding: None.

\section{Footnote}

Reporting Checklist: The authors have completed the ARRIVE reporting checklist. Available at http://dx.doi. org/10.21037/apm-21-65
Data Sharing Statement: Available at http://dx.doi. org/10.21037/apm-21-65

Conflicts of Interest: All authors have completed the ICMJE uniform disclosure form (available at http://dx.doi. org/10.21037/apm-21-65). The authors have no conflicts of interest to declare.

Etbical Statement: The authors are accountable for all aspects of the work in ensuring that questions related to the accuracy or integrity of any part of the work are appropriately investigated and resolved. This study was approved by the Hebei General Hospital Ethics Committee. (No.202103). All processes were performed according to the Guidelines for the Care and Use of Laboratory Animals of the Japanese Pharmacological Society.

Open Access Statement: This is an Open Access article distributed in accordance with the Creative Commons Attribution-NonCommercial-NoDerivs 4.0 International License (CC BY-NC-ND 4.0), which permits the noncommercial replication and distribution of the article with the strict proviso that no changes or edits are made and the original work is properly cited (including links to both the formal publication through the relevant DOI and the license). See: https://creativecommons.org/licenses/by-nc-nd/4.0/.

\section{References}

1. Liyanage $T$, Ninomiya $T$, Jha V, et al. Worldwide access to treatment for end-stage kidney disease: a systematic review. Lancet 2015;385:1975-82.

2. Whiting DR, Guariguata L, Weil C, et al. IDF Diabetes Atlas: Global estimates of the prevalence of diabetes for 2011 and 2030. Diabetes Res Clin Pract 2011;94:311-21.

3. Ogurtsova K, da Rocha Fernandes JD, Huang Y, et al. IDF Diabetes Atlas: Global estimates for the prevalence of diabetes for 2015 and 2040. Diabetes Res Clin Pract 2017;128:40-50.

4. Lewis EJ, Hunsicker LG, Clarke WR, et al. Renoprotective effect of the angiotensin-receptor antagonist irbesartan in patients with nephropathy due to type 2 diabetes. $\mathrm{N}$ Engl J Med 2001;345:851-60.

5. Brenner BM, Cooper ME, de Zeeuw D, et al. Effects of Losartan on Renal and Cardiovascular Outcomes in Patients with Type 2 Diabetes and Nephropathy. N Engl J Med 2001;345:861-9.

6. Deppe S, Böger RH, Weiss J, et al. Telmisartan: a review 
of its pharmacodynamic and pharmacokinetic properties. Expert Opin Drug Metab Toxicol 2010;6:863-71.

7. Weiss J, Sauer A, Divac N, et al. Interaction of angiotensin receptor type 1 blockers with ATP-binding cassette transporters. Biopharm Drug Dispos 2010;31:150-61.

8. Perkovic V, Jardine MJ, Neal B, et al. Canagliflozin and Renal Outcomes in Type 2 Diabetes and Nephropathy. N Engl J Med 2019;380:2295-306.

9. Heerspink HJL, Stefánsson BV, Correa-Rotter R, et al. Dapagliflozin in Patients with Chronic Kidney Disease. N Engl J Med 2020;383:1436-46

10. Mamidi RNVS, Dallas S, Sensenhauser C, et al. In vitro and physiologically-based pharmacokinetic based assessment of drug-drug interaction potential of canagliflozin. Br J Clin Pharmacol 2017;83:1082-96.

11. Syvänen S, Lindhe O, Palner M, et al. Species differences in blood-brain barrier transport of three positron emission tomography radioligands with emphasis on P-glycoprotein transport. Drug Metab Dispos 2009;37:635-43.

12. Ito K. ABCC2/Abcc2 transport property in different species and its modulation by heterogeneous factors. Drug Metab Pharmacokinet 2008;23:394-405.

13. Natarajan K, Xie Y, Nakanishi T, et al. Identification and characterization of the major alternative promoter regulating Bcrp1/Abcg2 expression in the mouse intestine. Biochim Biophys Acta 2011;1809:295-305.

14. Skrtić M, Yang GK, Perkins BA, et al. Characterisation of glomerular haemodynamic responses to SGLT2 inhibition in patients with type 1 diabetes and renal hyperfiltration. Diabetologia 2014;57:2599-602.

15. Cherney DZ, Perkins BA, Soleymanlou N, et al. Renal hemodynamic effect of sodium-glucose cotransporter 2 inhibition in patients with type 1 diabetes mellitus. Circulation 2014;129:587-97.

16. Health USDo, Human Services F, Drug a. Bioanalytical Method Validation, Guidance for Industry. Available online: https://www.fda.gov/media/70858/download. 2018.

17. Kaza M, Karaźniewicz-Łada M, Kosicka K, et al. Bioanalytical method validation: new FDA guidance vs. EMA guideline. Better or worse? J Pharm Biomed Anal 2019;165:381-5.

18. Alam P, Iqbal M, Foudah AI, et al. Quantitative determination of canagliflozin in human plasma samples using a validated HPTLC method and its application to a pharmacokinetic study in rats. Biomed Chromatogr 2020;34:e4929.

19. Iqbal M, Ezzeldin E, Al-Rashood KA, et al. Rapid determination of canagliflozin in rat plasma by UHPLCMS/MS using negative ionization mode to avoid adduct- ions formation. Talanta 2015;132:29-36.

20. Kobuchi S, Yano K, Ito Y, et al. A validated LC-MS/MS method for the determination of canagliflozin, a sodiumglucose co-transporter 2 (SGLT-2) inhibitor, in a lower volume of rat plasma: application to pharmacokinetic studies in rats. Biomed Chromatogr 2016;30:1549-55.

21. Dong ST, Niu HM, Wu Y, et al. Plasma Pharmacokinetic Determination of Canagliflozin and Its Metabolites in a Type 2 Diabetic Rat Model by UPLC-MS/MS. Molecules 2018;23:1229.

22. Mohamed D, Elshahed MS, Nasr T, et al. Novel LC-MS/ MS method for analysis of metformin and canagliflozin in human plasma: application to a pharmacokinetic study. BMC Chem 2019;13:82.

23. Wen J, Zeng M, Liu Z, et al. The influence of telmisartan on metformin pharmacokinetics and pharmacodynamics. J Pharmacol Sci 2019;139:37-41.

24. Tahara A, Takasu T, Yokono M, et al. Characterization and comparison of sodium-glucose cotransporter 2 inhibitors in pharmacokinetics, pharmacodynamics, and pharmacologic effects. J Pharmacol Sci 2016;130:159-69.

25. Choi MK, Nam SJ, Ji HY, et al. Comparative Pharmacokinetics and Pharmacodynamics of a Novel Sodium-Glucose Cotransporter 2 Inhibitor, DWP16001, with Dapagliflozin and Ipragliflozin. Pharmaceutics 2020;12:268.

26. Francke S, Mamidi RN, Solanki B, et al. In vitro metabolism of canagliflozin in human liver, kidney, intestine microsomes, and recombinant uridine diphosphate glucuronosyltransferases (UGT) and the effect of genetic variability of UGT enzymes on the pharmacokinetics of canagliflozin in humans. J Clin Pharmacol 2015;55:1061-72.

27. Stangier J, Schmid J, Türck D, et al. Absorption, metabolism, and excretion of intravenously and orally administered [14C] telmisartan in healthy volunteers. J Clin Pharmacol 2000;40:1312-22.

28. Chen X, Hu P, Vaccaro N, et al. Pharmacokinetics, Pharmacodynamics, and Safety of Single-Dose Canagliflozin in Healthy Chinese Subjects. Clin Ther 2015;37:1483-92.e1.

Cite this article as: Wang $\mathrm{XN}$, Li Y, Meng L, Ding CY, Dong ZJ. Evaluation of influence of telmisartan on the pharmacokinetics and tissue distribution of canagliflozin in rats and mice. Ann Palliat Med 2021;10(3):3086-3096. doi: 10.21037/ apm-21-65 